\title{
ANK2 Hypermethylation in Canine Mammary Tumors and Human Breast Cancer
}

\author{
Johannes J. Schabort ${ }^{1}$, A-Reum Nam ${ }^{1}$, Kang-Hoon Lee ${ }^{1}{ }^{\circledR}$, Seok Won Kim ${ }^{2}$, Jeong Eon Lee ${ }^{2}$ \\ and Je-Yoel Cho ${ }^{1, * \mathbb{D}}$ \\ 1 Department of Biochemistry, BK21 PLUS Program for Creative Veterinary Science Research \\ and Research Institute for Veterinary Science, College of Veterinary Medicine, Seoul National University, \\ 08826 Seoul, Korea; jan2schabort@snu.ac.kr (J.J.S.); arbjlvz@snu.ac.kr (A.-R.N.); khlee02@snu.ac.kr (K.-H.L.) \\ 2 Division of Breast Surgery, Department of Surgery, Samsung Medical Center, \\ Sungkyunkwan University School of Medicine, 81 Irwon-ro, Gangnam-gu, Seoul 06351, Korea; \\ seokwon1.kim@samsung.com (S.W.K.); jeongeon.lee@samsung.com (J.E.L.) \\ * Correspondence: jeycho@snu.ac.kr; Tel.: +82-02-880-1268
}

Received: 21 September 2020; Accepted: 16 November 2020; Published: 18 November 2020

\begin{abstract}
Canine mammary tumors (CMT) constitute the most common tumor types found in female dogs. Understanding this cancer through extensive research is important not only for clinical veterinary applications, but also in the scope of comparative oncology. The use of DNA methylation as a biomarker has been noted for numerous cancers in the form of both tissue and liquid biopsies, yet the study of methylation in CMT has been limited. By analyzing our canine methyl-binding domain sequencing (MBD-seq) data, we identified intron regions of canine ANK2 and EPAS1 as differentially methylated regions (DMGs) in CMT. Subsequently, we established quantitative methylation specific PCR (qMSP) of ANK2 and EPAS1 to validate the target hypermethylation in CMT tissue, as well as cell free DNA (cfDNA) from CMT plasma. Both ANK2 and EPAS1 were hypermethylated in CMT and highlighted as potential tissue biomarkers in CMT. ANK2 additionally showed significant hypermethylation in the plasma cfDNA of CMT, indicating that it could be a potential liquid biopsy biomarker as well. A similar trend towards hypermethylation was indicated in $\mathrm{HBC}$ at a specific $\mathrm{CpG}$ of the ANK2 target on the orthologous human region, which validates the comparative approach using aberrant methylation in CMT.
\end{abstract}

Keywords: CMT; hypermethylation; biomarker; cfDNA; HBC

\section{Introduction}

Canine mammary tumors (CMT) are the most common neoplasia diagnosed in female dogs and are found to be malignant in approximately $50 \%$ of cases [1-4]. Apart from the clear veterinary benefit to understanding the nature of this cancer more clearly, studying CMT can also be beneficial in the field of comparative oncology [5-7].

Dogs have been highlighted as excellent animal models for human cancer [5,6], and this includes the use of CMT as a comparative model for human breast cancer (HBC) [7-10]. The expedited study rate due to faster disease progression and shorter lifespan, histological and mechanistic similarities [5], spontaneous disease occurrence, and exposure to similar environments as humans make dogs ideal models for comparative oncology [11,12]. The similar environments to humans that dogs often inhabit as companion animals are particularly interesting from an epigenetic standpoint, as environmental stimuli have been known to influence epigenetics [13,14].

DNA methylation is an important method of epigenetic regulation and thus has often been studied in relation to cancer [15]. Aberrant global hypomethylation and localized, specific hypermethylation of 
tumor suppressors and hypomethylation of oncogenes are observed in cancer [16]. In CMT, aberrant methylation has been reported for a few genes such as ER $\alpha$ [17], DAPK1, MGMT [18], and BRCA1 [19], yet gene-specific methylation of CMT remains largely unknown. These methylation changes are often observed early during carcinogenesis [20] making them ideal biomarkers for early detection and prognosis. Tissue biomarkers remain the standard for cancer diagnosis and prognosis, yet in recent years the interest in liquid biopsy biomarkers have risen [21].

Liquid biopsies involve the investigation of cell-free DNA (cfDNA) found in the blood, plasma, serum, or other liquids from the body and often contain circulating tumor DNA. They are of clinical importance as they are less invasive and also allow for a more comprehensive view of the cancer biology as opposed to the view offered by tissue biopsies that is limited to a single site of the tumor at a single moment in the progression of the cancer [21]. Methylation of circulating cfDNA has been highlighted as a human cancer biomarker in liquid biopsies [22-24]. Previously, our lab has presented the cfDNA hypomethylation of LINE1 as a candidate liquid biopsy biomarker in both CMT and HBC, however, the method of restriction enzyme digestion followed by quantitative-PCR needs to be improved further for clinical use [25].

This study set out to identify and investigate differentially methylated regions (DMRs) in CMT, initially validating our findings in CMT tissues and then also in cfDNA isolated from plasma obtained from dogs with CMT with the intent of establishing candidate tissue and liquid biomarkers for cfDNA. Additionally, we correlated our differentially methylated target regions in our CMT data with HBC data to see if the differential methylation seen in these diseases is consistent across species at the targeted sites.

\section{Results}

\subsection{Identification of Differentially Methylated Regions}

Target hypermethylated DMRs were identified using MBD-seq and corresponding RNA-seq according to the scheme in Figure 1A. From our previous MBD-seq data obtained for 11 pairs of CMT and adjacent normal tissue from NCBI BioProject database (accession number PRJNA601533) [26], 16,061 differentially methylated genes (DMGs) were identified based on the presence of a differentially methylated region (DMR) within the gene body. To develop DNA methylation-based biomarkers applicable to CMT and HBC, we decided to narrow the scope to regions that contained a hypermethylated CpG island in CMT. A separate study could be performed to analyze the hypomethylated cohort. We further narrowed down the 1269 hypermethylated DMGs, based on the fold change (log2 fold change >1.5). Of these 20 identified targets, EPAS1, ANK2, DST and RUSC2 indicated a downregulation of gene expression in CMT noted from the RNA-seq data. Of these, ANK2 and EPAS1 were at a significant level ( $p$-value $<0.05$ ) and were thus selected for further analysis (Figure 1B). These identified CMT DMRs were found in CpG islands in the 21st and 1st introns of ANK2 and EPAS1, respectively, and the increase in methylation in the cancer samples are shown via linear mixed model (LMM) with thresholds of both $10 \%$ and $5 \%$ (Figure 1C). The overall methylation level detected via the MBD-seq for both target regions were shown to be significantly more methylated in the CMT samples as opposed to the normal samples (Figure 1D). Of the $11 \mathrm{CMT} /$ normal tissue pairs that were used for MBD-seq, 10 were also used in the RNA-seq analysis. These 10 pairs that had data for both MBD-seq and RNA-seq showed a general reverse correlation trend between expression and methylation for both targets, albeit not at a significant level (Figure 1E,F). Yet, the correlation plots and the associated density plots do clearly reveal that for both targets the level of methylation is higher in CMT than in normal, whereas the level of expression is lower. Compared to the paired normal samples an increase in methylation of ANK2 and EPAS1 along with a decrease in expression is indicated in 5/10 and 8/10 CMT samples, respectively (Figure 1E,F). Based on the accumulated bioinformatic data, comprising MBD-seq and RNA-seq data, ANK2 and EPAS1 were both highlighted as potential CMT biomarkers with DMRs in CMT and were chosen as the targets for the remainder of this study. 


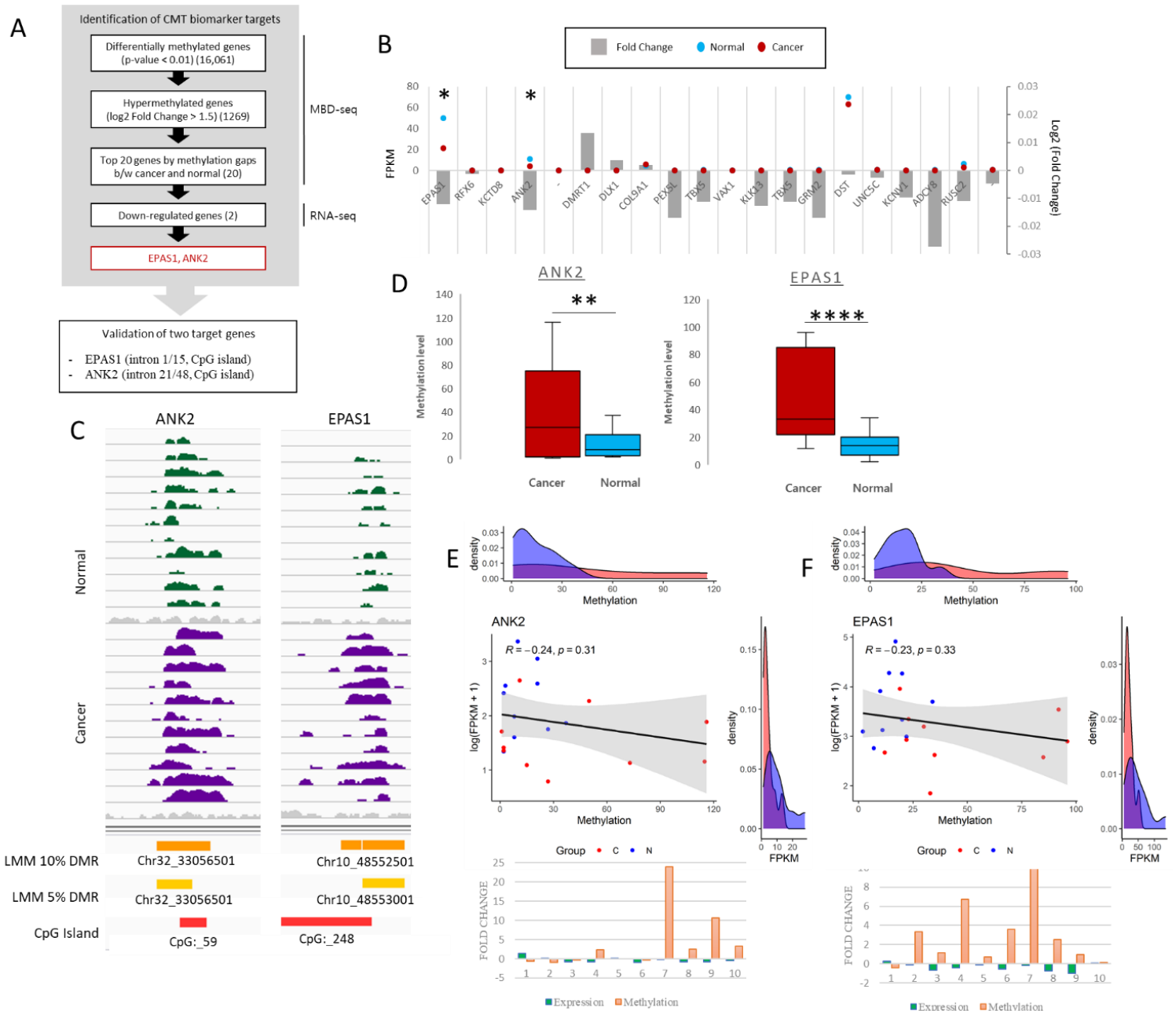

Figure 1. Selection of ANK2 and EPAS1 as canine mammary tumor (CMT) hypermethylated targets. (A) Schematic overview of target selection using MBD-seq and RNA-seq. Each step indicates the number of identified genes at the end of that step. (B) RNA-seq gene expression in FPKM of top 20 hypermethylated genes; blue dots $=$ normal, red dots $=\mathrm{CMT},{ }^{*}=p$-value $<0.05$. Fold change $(\log 2)$ for each target gene indicated with grey bar graph. (C) IGV peak calling for ANK2 and EPAS1 in both normal and cancer. Differential methylation assigned via LMM with both 5\% and 10\% threshold, and CpG island presence is indicated. (D) Overall methylation levels of ANK2 and EPAS1 from MBD-seq data for 11 paired CMT and adjacent normal samples. EdgeR. ${ }^{* *}=p$-value $<0.01,{ }^{* * * *}=p$-value $<0.0001$. (E) ANK2 and (F) EPAS1 correlation plots between expression (FPKM) and methylation of 10 paired CMT (red) and normal (blue) samples with matching density plots for both FPKM and methylation. Pearson correlation $|\mathrm{r}|$ value and $p$ value indicated. Fold change graphs are also depicted for 10 CMT samples indicating expression (green) and methylation (orange).

\subsection{Evaluation of Differentially Methylated Regions in CMT and Adjacent Normal Tissue}

The target intron regions of ANK2 and EPAS1 that were identified through MBD-seq were further analyzed to assess their differential methylation. Quantitative methylation specific PCR (qMSP) was chosen as the method by which methylation was to be analyzed, mainly due to its ability to quantify the methylation of numerous samples simultaneously without the need for exhaustive cloning and sequencing procedures. qMSP is furthermore a very sensitive technique that is able to distinguish a very small amount of methylated CpGs from unmethylated CpGs [27]. However, sequencing was still performed on three randomly selected pairs of CMT and normal gDNA for each target to establish a representative methylation pattern for each target and thus indicate in which areas to design the MSP primers (Figure 2). Both the ANK2 and EPAS1 target regions were methylated more in the CMT than 
the paired normal samples, although the amount of differential methylation varied across the sample pairs (Figure 2).

A

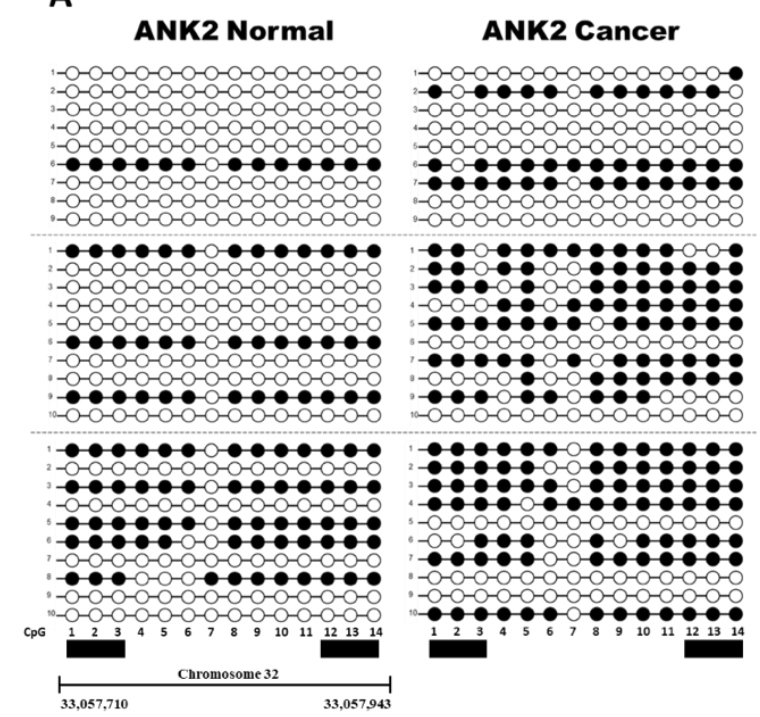

B

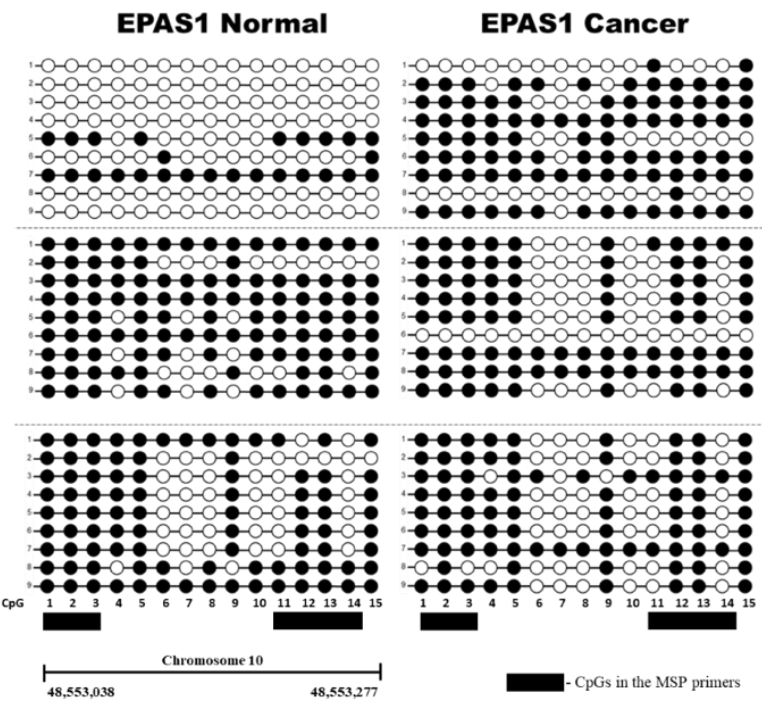

Figure 2. Bisulfite Sequencing PCR (BSP) of ANK2 and EPAS1. Between 9 and 10 colonies were sequenced for three paired normal and CMT samples (separated by grey dotted line) for (A) ANK2 and (B) EPAS1. Methylation of a particular CpG is indicated by a black circle, and non-methylation is indicated by a white circle. The CpGs that were included in the forward and reverse MSP primers for each respective target with a black box.

qMSP was then performed to investigate more sample pairs. The Methylation Index is presented and is based on the demethylation index first introduced by Akirav et al. [28], with the adjustment of measuring the amount of methylated DNA as opposed to unmethylated DNA. This method uses bisulfite sequencing PCR (BSP) primers that flank the MSP region of interest to normalize the MSP readings (Figure $3 \mathrm{~A}$ ). Of the 15 sample pairs analyzed for the ANK2 target, 12 were more methylated in CMT compared to normal based on the methylation index, whereas nine out of 15 EPAS1 target samples were more methylated in CMT than in paired normal (Figure 3B). The overall methylation was shown to be significantly more methylated in CMT for both the ANK2 and EPAS1 targets, based on paired t-tests (Figure 3C). Receiver operating characteristic (ROC) curves were constructed for both targets to access the sensitivity and specificity of using CMT hypermethylation as biomarkers. ANK2 had an area under the curve (AUC) of 0.764 and EPAS1 had an AUC of 0.733 (Figure 3D). Overall, the quantitative MSP results validated what was shown in the MBD and sequencing data; the targeted ANK2 intron 21 and EPAS1 intron 1 regions are hypermethylated in CMT and are candidate tissue biomarkers for this disease. 

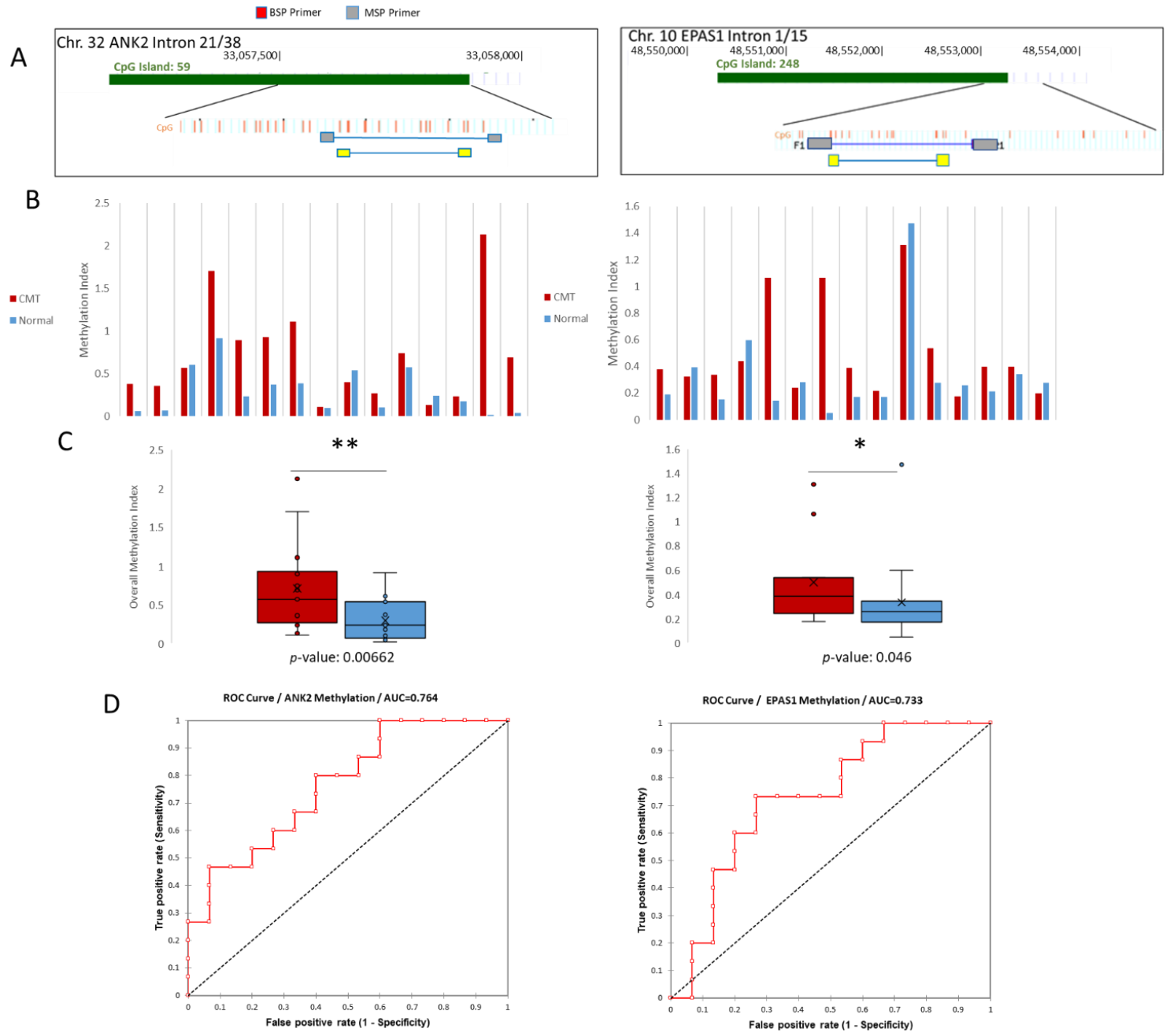

Figure 3. Quantitative Methylation Specific PCR (qMSP) validation of ANK2 and EPAS1 hypermethylation. (A) Schemes for both ANK2 and EPAS1 indicating the target region in terms of its position on the CPG island of its respective intron. BSP primers (grey) are shown to flank the regions targeted by the MSP primers (yellow). (B) Methylation Indexes for both ANK2 and EPAS1. CMT samples are shown in red and paired normal samples are shown in blue. (C) Overall methylation indexes for both ANK2 and EPAS1 are shown. CMT in red, normal in blue. ${ }^{*}=p$-value $<0.05$, ** $=p$-value $<0.01$. (D) ROC curve analyses for ANK2 and EPAS1.

\subsection{Detection of Differential Methylation in Canine Plasma cfDNA}

qMSP has been utilized in previous studies to detect methylation of cfDNA [29,30]. One study found a significant agreement between ESR1 methylation of paired plasma and primary ovarian cancer tumors [31]. To evaluate whether the hypermethylation trends noted in CMT tissue could also be detected in cfDNA and thus serve as potential liquid biopsy biomarkers for CMT, we conducted the same qMSP procedure on cfDNA isolated from plasma samples of CMT and normal female dogs. The ANK2 target was analyzed in pooled cfDNA samples from 19 CMT dogs and 10 normal dogs. The hypermethylation trend of the ANK2 target region in CMT extended to cfDNA as is shown by the significantly higher methylation index (Figure 4A). Interestingly, the EPAS1 target, which was investigated in cfDNA samples from $10 \mathrm{CMT}$ and 10 normal dogs, did not indicate an increased level of methylation for CMT as was seen in the tissue samples (Figure 4B). In contrast, the CMT cfDNA showed less methylation in CMT than normal plasma for EPAS1. Overall, in canine cfDNA, ANK2 demonstrated significant hypermethylation in CMT samples, which proposes it as a potential liquid biopsy biomarker for CMT. 
A

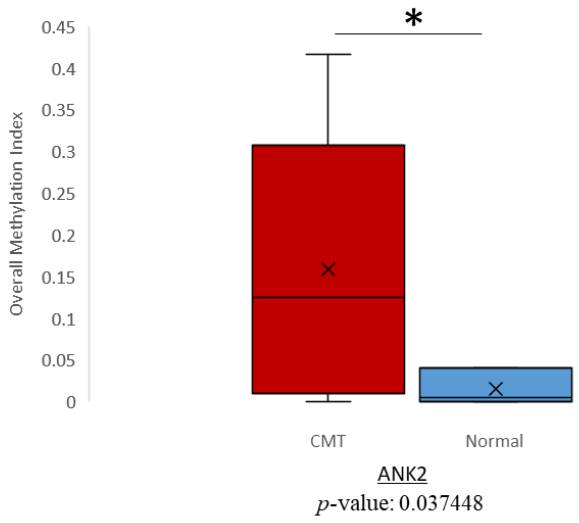

B

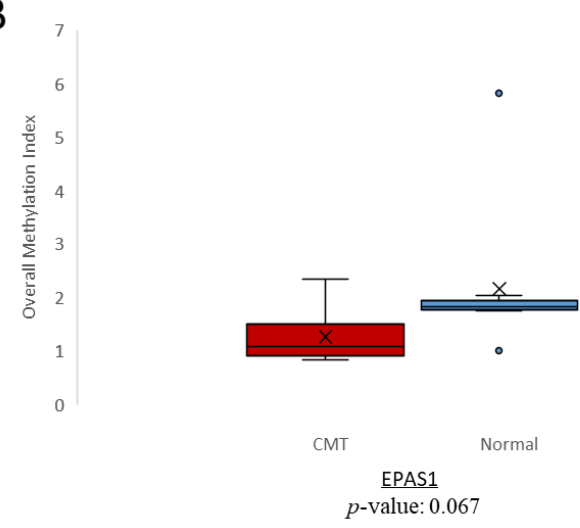

Figure 4. Canine Mammary Tumor (CMT) hypermethylation analysis in cfDNA. Overall methylation index for (A) ANK2 and (B) EPAS1 in CMT (red) and normal (blue) cfDNA. ${ }^{*}=p$-value $<0.05$.

\subsection{Orthologous Human Regions Analyzed from TCGA Data}

To investigate whether the hypermethylation trend seen at the target regions in CMT would correspond to what is seen in HBC, we mapped the target regions found on the dog genome (CanFam3.1) to the human genome (HG19). This indicated that our $500 \mathrm{bp}$ regions identified from the canine MBD-seq for the ANK2 and EPAS1 targets had orthologous human regions of $405 \mathrm{bp}$ with $79 \%$ sequence identity and $221 \mathrm{bp}$ with $76 \%$ sequence identity, respectively (Supplementary Table S1). The EPAS1 target had an orthologous region that was also located in a CpG island at the $3^{\prime}$ end of the first intron, just as in canines. The ANK2 target, which is situated in the 21st intron of the canine gene, similarly had an orthologous region in the 21st intron of human ANK2, however, this region does not contain a CpG island on the human genome, which makes it unsuitable for study with the qMSP method that we employed in this study. Even so, we investigated data from The Cancer Genome Atlas (TCGA) for the target regions using Wanderer [32] and found ANK2 to be hypermethylated at three of the four CpG probes in this region (cg 25915539, cg17665652, and cg08448479), which corresponds to the hypermethylation that we observed in the orthologous region in dog (Figure 5A). Interestingly, even though the amount of $\mathrm{CpGs}$ in this region is much less in humans than dogs, these three hypermethylated human $\mathrm{CpGs}$ are all conserved between the two species and were shown to be hypermethylated in the CMT dog samples (Figure 2A). The expression data from TCGA also indicated a downregulation of $A N K 2$ in $\mathrm{HBC}$, which matches our canine data (Figure $5 \mathrm{~B}$ ), and these data are further supported by the survival plot of ANK2 that shows a lower survival rate with decreased ANK2 expression (Figure 5C). The human EPAS1 region orthologous to the hypermethylated canine EPAS1 region that we analyzed contained 2 hypermethylated $\mathrm{CpG}$ probes according to the TCGA data (Figure 5D). Furthermore, the EPAS1 expression level was shown to be downregulated in HBC and the survival rate was decreased in accordance with EPAS1 downregulation (Figure 5E,F).

To evaluate whether these trends would hold for clinical cfDNA samples, we isolated some from human plasma, obtained from both normal and HBC patients, and subjected it to bisulfite PCR sequencing. Unexpectedly, the EPAS1 target region did not show any methylation in this region for either HBC (four samples) or normal (two samples consisting of five and six pooled cfDNA isolates, respectively) [33], but this does coincide with the unexpected lack of cfDNA hypermethylation in CMT (Figure 4B). The hypermethylation trends shown in EPAS1 CMT tissue and HBC data from TCGA seems to not hold up in either canine or human cfDNA. On the other hand, sequencing results of the ANK2 target region from human cfDNA was more in line with what was suggested by the TCGA data. We sequenced colonies from four separate samples of HBC cfDNA and from 5 pooled normal cfDNA samples. Of the three CpGs indicated to be hypermethylated in this region from the TCGA data, only cg08448479, designated as CpG 5, was also shown to be more methylated in HBC cfDNA according to our sequencing results (Figure 5G). CpG 5 showed an increase in methylation from $71.88 \%$ 
in normal to $85.48 \%$ in $\mathrm{HBC}$, which is consistent with the largest increase in methylation seen for the orthologous canine CpGs from 31.04\% in normal to 62.07\% in CMT (Figure 5G). We attempted to perform the qMSP procedure on pooled samples of HBC cfDNA (14 samples) and pooled sample of normal human cfDNA (5 samples) with MSP primers that incorporated CpGs 1, 2, and 3 and CpGs 5 and 6 in the forward and reverse primers, respectively, but no significant difference was observed (Supplementary Figure S1). In both the TCGA dataset and the cfDNA sequencing, the ANK2 target indicated hypermethylation, even if limited to only a few CpGs, which is concurrent with what was seen in the canine CMT data.

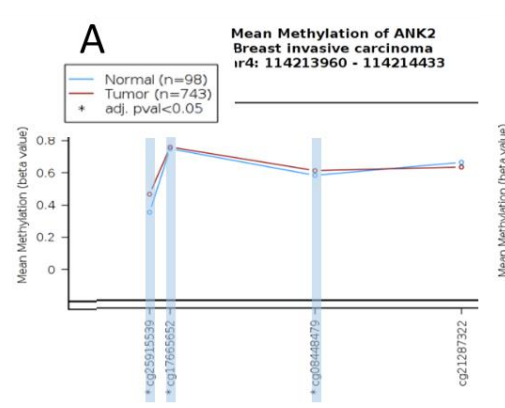

B
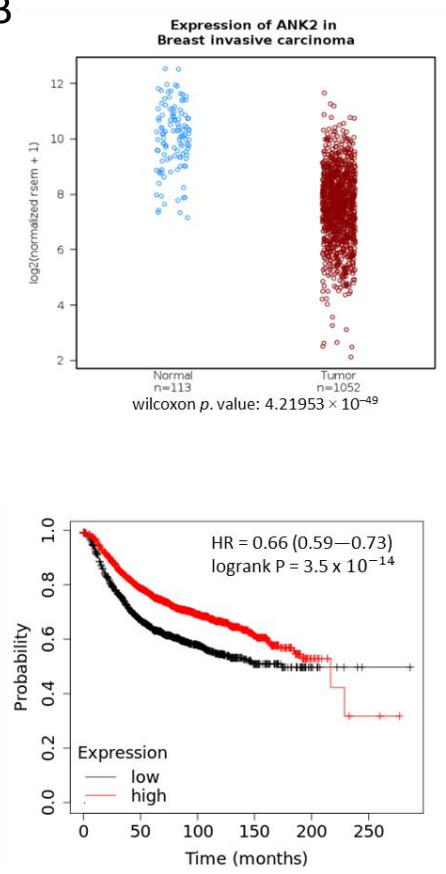

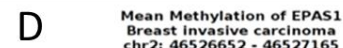

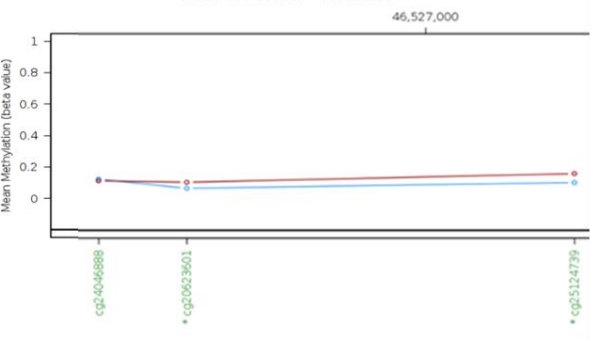

E
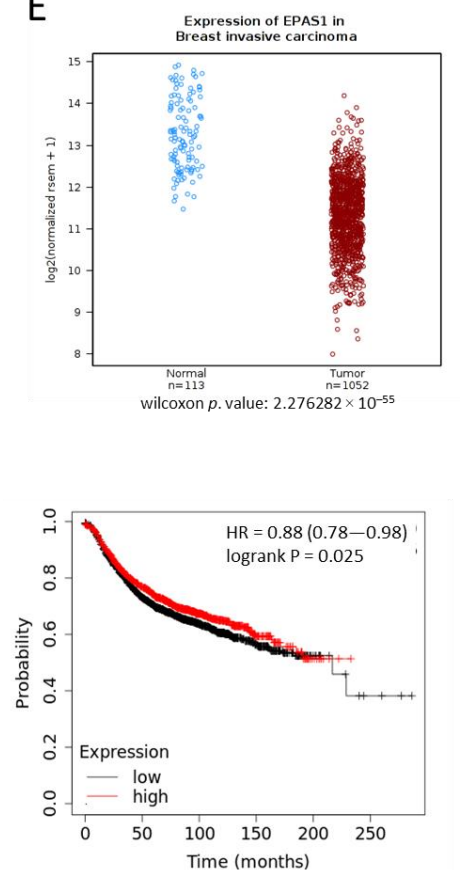

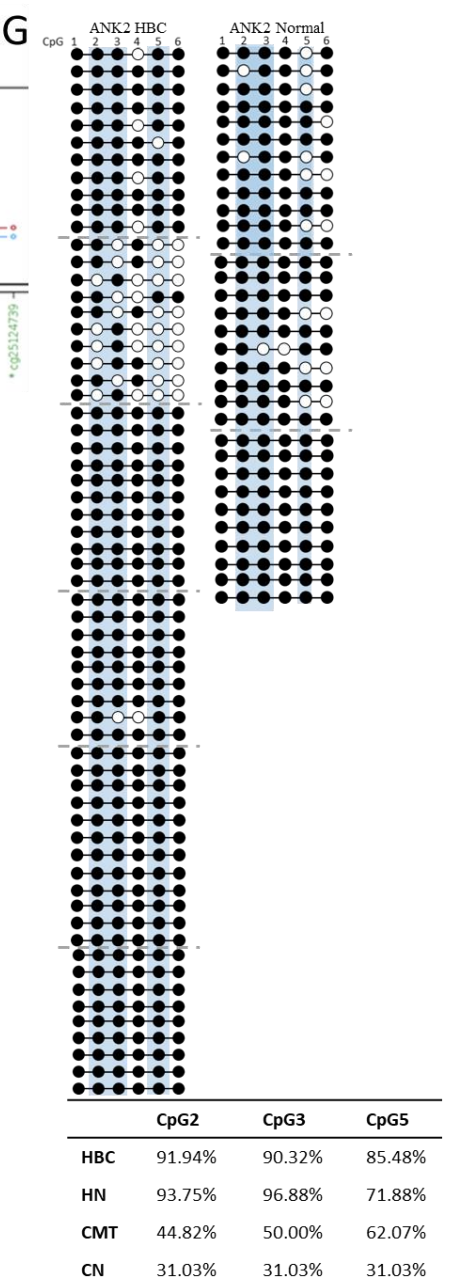

Figure 5. Hypermethylation of orthologous human target ANK2 and EPAS1 regions. Mean Methylation (beta value) plots for orthologous human (A) ANK2 and (D) EPAS1 regions for normal (blue) and invasive breast cancer patient(red) data obtained from TCGA. Specific methylation probes, annotated at the bottom of the graph are indicated to be in a CpG island when written in green (only seen for EPAS1), and designated as significant $\left(^{*}\right)$ with an adjusted $p$-value $<0.05$. Three significantly hypermethylated CpGs from ANK2 are highlighted in light blue to indicate that they are conserved in the dog genome. (B) ANK2 and (E) EPAS1 log2 (normalized rsem +1) expression data from TCGA for normal (blue) and invasive breast cancer patients (red). Wilcoxon $p$-values are indicated. (C) ANK2 and (F) EPAS1 Kaplan-Meier plots indicting relapse-free survival with high and low expressions each. (G) ANK2 sequencing results for cfDNA from normal and HBC patients. Between 10 and 11 colonies were sequenced for 6 individual HBC cfDNA samples (separated by grey dotted lines) and between 10 
and 12 colonies were sequenced from 3 separate normal cfDNA samples. Methylation of a specific CpG is indicated by a black circle and non-methylation by a white circle. The CpGs at positions 2, 3 , and 5 are highlighted in light blue as these are the CpGs that correlate with the hypermethylated $\mathrm{CpG}$ probes indicated in (A) and the respective percentage methylation for these CpGs in the HBC and normal human (HN) cfDNA samples, as well as in canine CMT and normal (CN) tissue (Figure 2A), are indicated in the inserted table.

\section{Discussion}

CMT is a common malignancy in female dogs and, moreover, in the scope of using dogs as animal models for human disease, its study can be translated to HBC. The field of comparative oncology using canine models have been increasing in popularity, yet the epigenome of canines with CMT remains incomplete. Methylation of DNA has been highlighted as a biomarker in both tissue and liquid biopsies [22-24], and thus we set out in this study to evaluate potential methylation biomarkers in CMT. This study identified novel regions in the 21st and 1st introns of ANK2 and EPAS1, respectively, that were significantly hypermethylated in CMT tissue and, in the case of ANK2, also in cfDNA isolated from dogs suffering from the disease. The ANK2 region was furthermore shown to be hypermethylated in human cfDNA at a corresponding $\mathrm{CpG}$ on the orthologous human region.

ANK2 encodes multiple AnkyrinB isoforms, which are integral membrane proteins that connect the lipid bilayer to the membrane skeleton [34]. In this present study, it was indicated that ANK2 mRNA expression was downregulated in CMT and also in HBC based on TCGA data. It is of note that other members of the ankyrin family, namely ANK1 and ANK3, have been shown to be overexpressed in multiple human cancers including breast cancer [35,36], while ANK2 overexpression has been shown in pancreatic cancer where it contributed to the malignant phenotype [37]. However, other studies found that ANK2 gene expression was downregulated in colorectal cancer [38] and thyroid cancer [39], which corresponds to what we found in this study. The expression status and role of ANK2 seems to vary amongst cancer types, and further investigation in a larger variety of cancers is required to better understand its relationship to cancer. Moreover, the role of cancer associated aberrant methylation in ANK2 remains largely unknown. One previous study compiled datasets for nasopharyngeal cancer and found ANK2 to be hypomethylated and upregulated, highlighting it as a potential biomarker for this cancer type [40]. Our study found a hypermethylated intron 21 region of ANK2, in both tissue and cfDNA, and it was highlighted as a potential tissue and liquid biopsy biomarker for CMT. Yet, the impact of this specific methylation in CMT as well as the fuller extent of ANK2 methylation, on the promotor region for instance, remains unknown and should be studied further. Furthermore, the orthologous human region does contain hypermethylated CpGs but outside of a CpG island which makes the qMSP method difficult, even more so when considering that of the six CpGs in the region only one seemed to be differentially methylated based on the sequencing data in Figure 5G. The subsequent failure of the qMSP in distinguishing significantly between the HBC and Normal ANK2 is probably due to there only being one $\mathrm{CpG}(\mathrm{CpG}$ ) that is differentially methylated, and the technique is reliant on more than one $\mathrm{CpG}$ being differentially methylated. Additional sequencing of this region in more samples would be the best way to highlight the differential methylation in the respective CpGs. Other regions on ANK2 that contain conserved CpG islands could potentially be more successfully analyzed via the qMSP method and could potentially prove more influential in the downregulation of expression seen in Figure 5B. Pyrosequencing could be another alternative to pursue, as opposed to cloning and Sanger sequencing. We did not observe any methylation difference on the promoter region in our previous MBD-seq data [26]. This orthologous human region should be further studied in the future in tissue and cell culture to further investigate the nature and role of these hypermethylated CpGs play a significant role in HBC. To our knowledge, no other study yet been able to elucidate the state of ANK2 in either HBC or CMT, which makes our novel investigation of ANK2 in these diseases very insightful.

EPAS1, on the other hand, is relatively well studied in its relation to cancer, including breast cancer. EPAS1 is part of the hypoxia inducible factor (HIF) family and plays an important role in the hypoxic 
response pathway which is often associated with tumorigenesis [41]. EPAS1 acts as a transcription factor in hypoxic conditions with many of its targets being known to contribute to carcinogenesis, such as VEGF and VEGF-R1 [41,42]. It has been highlighted as an oncogene in numerous cancer types $[43,44]$. However, EPAS1 has also been described as tumor suppressive in various other cancer types including neuroblastoma, colon cancer, and hepatocellular carcinoma, and shown to reduce tumor growth and improve patient outcomes when overexpressed [45-48]. In breast cancer, the role and expression levels of EPAS1 have been indicated to be subtype specific. Klahan et al. [49] found EPAS1 to be a subtype specific lymphovascular invasion marker in ER- and HER2+ breast cancer. It has additionally been demonstrated that there is an estrogen dependent downregulation of EPAS1 in ER+ but not ER- HBC and proposed EPAS1 as a negative prognostic marker in HER2+ invasive breast cancer, since overexpression of EPAS1 worsen the prognosis in HER2 and ER+ [50,51].

This present study indicated an overall downregulation of EPAS1 in CMT and in invasive HBC, yet the role of DNA methylation still remains unclear as, despite the indication of hypermethylation of the proposed region in the first intron of human EPAS1 shown in the TCGA data, the cfDNA analysis showed no methylation difference between normal and HBC patients. Similarly, the canine cfDNA data did not show any increased methylation in this region. However, significant hypermethylation was noted in CMT tissue compared to paired adjacent normal tissue. This discrepancy between the tissue methylation and cfDNA methylation could potentially be explained by a lesser abundance of circulating tumor DNA (ctDNA) compared to normal cfDNA isolated from the plasma samples used, seeing as even though tumors usually secrete a larger amount of cfDNA in the plasma there is currently no ideal method for separating ctDNA from total cfDNA. ctDNA is furthermore highly fragmented which could hinder its detection [23]. Analyzing the methylation of the EPAS1 target region in circulating tumor cells (CTCs) could prove insightful. The hypermethylation and mRNA downregulation noted in the CMT tissue is however concurrent with previous studies where EPAS1 has been shown to be hypermethylated in its promotor with downregulated mRNA expression in non-small cell lung cancer [52] and colon cancer [47]. Although a separate study showed EPAS1 to be hypomethylated in colon cancer [53]. In CMT tissue, our study of EPAS1 and its indications of hypermethylation in cancer as opposed to normal remains a novel and intriguing finding and highlights this target as a candidate tissue biomarker for CMT. Yet, future studies should endeavor to delve deeper into the methylation of the targeted intron region in $\mathrm{HBC}$ by analyzing tissue and cell culture samples. Elucidating the functional role of this hypermethylation in CMT and potentially HBC, specifically in ER+ subtypes that have previously indicated downregulation in HBC [50] and in HER2 subtypes, where it has been implicated as a prognostic marker [51], is important to understanding the regulation of EPAS1 and its potential role in CMT and HBC.

Overall, this study set out to investigate CMT tissues and cfDNA isolated from plasma obtained from dogs with CMT for potential biomarkers and attempt to translate the findings to HBC by ways of comparative oncology. This study identified novel hypermethylated intron regions for ANK2 and EPAS1 that could potentially serve as tissue biomarkers for CMT. Furthermore, the ANK2 target also showed hypermethylation in cfDNA isolated from CMT, which highlights it as a potential liquid biopsy biomarker for CMT. In HBC, one CpG from the orthologous human ANK2 region likewise indicated a hypermethylated trend in cfDNA isolated from HBC patients, which emphasizes the link between CMT and HBC epigenetic regulation.

\section{Materials and Methods}

\subsection{Ethics}

This study, including the materials and methods, was reviewed and approved by the Seoul National University Institutional Review Board/Institutional Animal Care and Use Committee (IACUC\# SNU-170602-1, 02 June 2017/IRB\#SNU 16-10-063, 6 October 2016), Samsung Medical Center IRB 
(\#SMC2016-07-129-015, 12 July 2016), and the Seoul Metropolitan Government-Seoul National University Boramae Medical Center IRB (\#20161123/16-2016-99/121, 23 November 2016).

\subsection{Tissue and Plasma Samples}

Tissue samples used in this study consisted of surgically removed CMT tissue and paired adjacent normal tissue obtained from a variety of dog breeds. Tumors identified as carcinomas were selected, yet the selection was not subtype specific (simple, complex, and ductal carcinomas) seeing as the MBD-seq data (accession number PRJNA601533) [26] did not show any subtype specific differences in methylation patterns for our targets. Canine blood samples were taken from dogs diagnosed with mammary carcinoma and normal samples were obtained from healthy dogs without cancer. A detailed roster of the dogs used can be found in Supplementary Table S2. Depending on the dog size, $2-4 \mathrm{~mL}$ of blood was collected in Vacuette EDTA tubes (Greiner Bio-One, Kremsmunster, Austria).

Human blood samples were obtained from Samsung Medical Center, Seoul. After written informed consent was given, $4-6 \mathrm{~mL}$ of human blood was obtained from HBC patients scheduled to undergo surgery and from healthy control subjects that indicated no breast abnormalities upon examination. A detailed roster of the human subjects can be found in Supplementary Table S3.

Both canine and human plasma samples were separated from the whole blood immediately after blood collection. An equal volume of Ficoll-Paque PLUS (GE Healthcare, Orsay, France) was added to each blood sample and centrifuged for $30 \mathrm{~min}$, at $500 \times \mathrm{g}, 18^{\circ} \mathrm{C}$ without brake. Plasma was collected from the supernatant and stored at $-80^{\circ} \mathrm{C}$ until use.

\subsection{Correlation Analysis between Methylation and Gene Expression}

We performed an integrative analysis of MBD-seq (accession number PRJNA601533) [26] and RNA-seq data (SRA accession number: SRR8741587-SRR8741602) [25] from 8 overlapped tissue samples to identify canine mammary gland DNA methylation markers. We first selected DMRs which are located in a CPG island on the gene body and examined the correlation between DNA methylation and gene expression for each sample. To inspect the impact of DNA methylation on the local regulation of gene expression, the Pearson correlation ( $r$ ) was calculated between the read count for DMRs located in $\mathrm{CpG}$ regions and the expression values of the corresponding genes. $\log (\mathrm{fpkm}+1)$ values were used to avoid taking $\log 0$ in the case of there being 0 counts. $|r|>0.3$ and an adjusted $p$-value $<0.05$ were set as the cutoffs for a significant correlation.

\subsection{DNA Isolation}

Genomic DNA was isolated from $25 \mathrm{mg}$ samples of paired CMT and adjacent normal tissues using the DNEasy Blood \& Tissue kit (Qiagen, Hilden, Germany) according to the manufacturer's protocol. The recovered gDNA was quantified using a nanodrop spectrophotometer. Circulating cfDNA was isolated from both human (Normal and Breast Cancer patients) and canine (Normal and CMT patients) plasma samples using the QIAamp Circulating Nucleic Acid Kit (Qiagen, Hilden, Germany) according to the manufacturer's instructions. In short, $500 \mathrm{uL}$ of plasma was brought up to $1 \mathrm{~mL}$ with PBS and lysed with proteinase $\mathrm{K}$ and Buffer ACL before being bound, washed, and eluted from QIAamp mini columns on a vacuum manifold. Recovered cfDNA was quantified with a Qubit 3.0 Fluorometer using the Qubit HS dsDNA Assay (Invitrogen, Carlsbad, CA, USA), according to the manufacturer's protocol.

\subsection{Bisulfite Sequencing}

DNA samples were Bisulfite treated using the EZ DNA Methylation-Lightning kit (Zymo Research, Irvine, CA, USA), according to the manufacturer's instructions. Bisulfite treated DNA was quantified using the Qubit ssDNA Assay on the Qubit 3.0 Fluorometer (Invitrogen, Carlsbad, CA, USA). Paired samples were brought to equal concentrations by diluting with water as appropriate before qMSP was performed. 
Randomly selected samples of Bisulfite treated gDNA was sequenced to ascertain what the general methylation patterns are for both the ANK2 and EPAS1 targets in both CMT and normal tissue. Bisulfite Sequencing PCR (BSP) was conducted using the BSP primers (Supplementary Table S4) and HotStart Taq DNA Polymerase (Bioneer, Daejeon, Korea). Successful amplification was validated via gel electrophoresis on $2.0 \%$ Agarose gels. PCR amplicons were purified using the MEGAquick-spin Plus Total Fragment DNA Purification kit (Intron Biotechnology, Seongnam, Korea) according to the manufacturer's protocol. The amplicons were then ligated into pGEM T-Easy vector (Promega, Madison, WI, USA) and transformed into competent E. coli cells via heat-shock at $45^{\circ} \mathrm{C}$ for $45 \mathrm{~s}$ and plated on LB Agar plates that had been treated with Ampicillin, X-Gal, and IPTG to allow for Blue/White screening of colonies. White colonies were picked and sent for Sanger sequencing (Macrogen, Seoul, Korea). cfDNA from human samples, HBC and normal, were similarly subjected to BSP sequencing.

\subsection{Quantitative Methylation-Specific PCR (qMSP)}

To investigate the methylation of both cancer and normal gDNA and cfDNA, quantitative MSP was performed on each sample with MSP primers (Supplementary Table S4) using the CFX96 Real-time PCR Detection System (Bio-Rad Laboratories, Hercules, CA, USA). The same samples were also subjected to quantitative PCR using the BSP primers (Supplementary Table S4), which were designed to flank the region of interest, to normalize the MSP readings and allow for the calculation of the Methylation Index, which was based on the demethylation index first introduced by Akirav et al. [28]. Each qMSP reaction contained 0.5 units Hotstart Taq DNA Polymerase (Bioneer, Daejeon, Korea), $0.625 \mathrm{mM} \mathrm{MgCl}$, $0.2 \mathrm{mM}$ dNTPs, 0.5X SYBER Green (Life Technologies, Carlsbad, CA, USA) and 10 pmol each of forward and reverse primers in a $20 \mu \mathrm{L}$ total volume. For the gDNA samples, 5-30 ng of template DNA was added, whereas 120-1200 pg was added for the cfDNA samples. Each sample was run in triplicate using both the MSP and BSP primer sets and the thermal cycler conditions were as follows: $95^{\circ} \mathrm{C}$ for $15 \mathrm{~min}$, denaturation at $95^{\circ} \mathrm{C}$ for $30 \mathrm{~s}$, annealing at Annealing Temperature (Supplementary Table S4) for $30 \mathrm{~s}$, elongation at $72{ }^{\circ} \mathrm{C}$ for $30 \mathrm{~s}$ and final elongation at $72{ }^{\circ} \mathrm{C}$ for $5 \mathrm{~min}$.

All primers were manually designed and checked using the OligoEvaluator ${ }^{\mathrm{TM}}$ webtool. MSP primers were designed with at least $6 \mathrm{CpGs}$ included in the set of primers, and with at least one CpG located in the last 3 bases at the $3^{\prime}$ ends of the primer. BSP primers were designed with at least 4 non-CpG C's included in the primer set. For canine primer optimization, there are not any universally methylated and unmethylated controls commercially available. In-house controls were thus made. In short, fully methylated canine controls were made by treating $\sim \mu \mathrm{g}$ of canine gDNA with MssI methyltransferase (New England Biolabs, Ipswich, MA, USA) according to the manufacturer's protocol. Fully unmethylated DNA was made by performing PCR with primers that flank the region of interest. The resulting amplimers were thus devoid of any methylation and served as completely unmethylated control template. Human primers were optimized on fully methylated and fully unmethylated human HCT116 DKO DNA (Zymo Research, Irvine, CA, USA).

\subsection{Human TCGA Data}

Using liftOver [54], we were able to convert the canine (CanFam3.1) genome coordinates of the differentially methylated intron regions of ANK2 and EPAS1 that we identified from the MBD-seq data to their orthologous regions on the human genome (Hg38). BLAST (https://blast.ncbi.nlm.nih.gov) was used to align the nucleotide sequences and determine the amount of sequence homology.

HBC data for the ANK2 and EPAS1 intron targets was obtained using Wanderer [32], which utilizes 450k Infinium Chip methylation arrays and Illumina HISeq RNA-seq data from TCGA. Survival plots were generated using Kaplan-Meier Plotter with the auto selected cutoff enabled, which utilizes the expression data for the genes of interest, ANK2 (202920_at) and EPAS1 (200878_at), and the relapse free survival data of 3951 patients obtained from the GEO database [55]. The Kaplan-Meier Plotter 
software groups were expressed as high or low according to the median expression and then a Kaplan-Meier plot was used to compare the two groups [55].

Supplementary Materials: Supplementary materials can be found at http://www.mdpi.com/1422-0067/21/22/ 8697/s1.

Author Contributions: J.J.S. mainly performed experiments and analysis, and wrote the first draft of the manuscript; A.-R.N. conducted the MBD-Seq and RNA-Seq, and assisted in writing the manuscript; K.-H.L. provided scientific discussion and assisted in analysis and writing the manuscript; S.W.K. and J.E.L. provided samples and scientific discussion; J.-Y.C. conceived and developed the study, provided scientific discussion, and revised the final manuscript. All authors have read and agreed to the published version of the manuscript.

Funding: This research was supported by the Bio \& Medical Technology Development Program of the National Research Foundation (NRF) funded by the Ministry of Science and ICT (\#2016M3A9B6026771 and \#2014M3A9D5A01073598).

Acknowledgments: We would also like to thank Wan-Hee Kim from the College of Veterinary Medicine, Seoul National University, Bumjo Oh from the Seoul Metropolitan Government-Seoul National University Boramae Medical Center, and Soo-Youn Lee from Samsung Medical Center, Sungkyunkwan University, for providing clinical specimens and additional scientific discussion.

Conflicts of Interest: The authors declare no competing interests.

\section{References}

1. Moe, L. Population-based incidence of mammary tumours in some dog breeds. J. Reprod. Fertil. Suppl. 2001, $57,439-443$.

2. Sorenmo, K.U. Canine mammary gland tumors. Vet. Clin. N. Am. Small Anim. Pract. 2003, 33, 573-596. [CrossRef]

3. Salas, Y.; Márquez, A.; Diaz, D.; Romero, L. Epidemiological Study of Mammary Tumors in Female Dogs Diagnosed during the Period 2002-2012: A Growing Animal Health Problem. PLoS ONE 2015, 10, e0127381. [CrossRef]

4. Sleeckx, N.; de Rooster, H.; Kroeze, E.J.B.V.; van Ginneken, C.; van Brantegem, L. Canine Mammary Tumours, an Overview. Reprod. Domest. Anim. 2011, 46, 1112-1131. [CrossRef]

5. Paoloni, M.; Khanna, C. Translation of new cancer treatments from pet dogs to humans. Nat. Rev. Cancer 2008, 8, 147-156. [CrossRef]

6. Rowell, J.L.; McCarthy, D.O.; Alvarez, C.E. Dog models of naturally occurring cancer. Trends Mol. Med. 2011, 17, 380-388. [CrossRef]

7. Gray, M.E.; Meehan, J.; Martínez-Pérez, C.; Kay, C.; Turnbull, A.K.; Morrison, L.R.; Pang, L.Y.; Argyle, D. Naturally Occurring Canine Mammary Tumors as a Translational Model for Human Breast Cancer. Front. Oncol. 2020, 10, 617. [CrossRef]

8. Strandberg, J.D.; Goodman, D.G. Animal model of human disease: Canine mammary neoplasia. Am. J. Pathol. 1974, 75, 225-228.

9. Visan, S.; Balacescu, O.; Berindan-Neagoe, I.; Catoi, C. In vitro comparative models for canine and human breast cancers. Clujul. Med. 2016, 89, 38-49. [CrossRef]

10. Abdelmegeed, S.M.; Mohammed, S. Canine mammary tumors as a model for human disease. Oncol. Lett. 2018, 15, 8195-8205. [CrossRef]

11. Wartenberg, J.A.B.D. Environmental causes for sinonasal cancers in pet dogs, and their usefulness as sentinels of indoor cancer risk. J. Toxicol. Environ. Health Part A 1998, 54, 579-591. [CrossRef]

12. Lin, C.-H.; Lo, P.-Y.; Wu, H.-D.; Chang, C.; Wang, L.-C. Association between indoor air pollution and respiratory disease in companion dogs and cats. J. Vet. Intern. Med. 2018, 32, 1259-1267. [CrossRef]

13. Bollati, V.; Baccarelli, A. Environmental epigenetics. Heredity 2010, 105, 105-112. [CrossRef]

14. Baccarelli, A.; Bollati, V. Epigenetics and environmental chemicals. Curr. Opin. Pediatr. 2009, 21, 243-251. [CrossRef]

15. Pfeifer, G.P. Defining Driver DNA Methylation Changes in Human Cancer. Int. J. Mol. Sci. 2018, 19, 1166. [CrossRef]

16. Baylin, S.B.; Jones, P.A. Epigenetic Determinants of Cancer. Cold Spring Harb. Perspect. Biol. 2016, 8, a019505. [CrossRef] 
17. Brandão, Y.D.O.; Toledo, M.B.; Chequin, A.; Cristo, T.G.; Sousa, R.S.; Ramos, E.A.S.; Klassen, G. DNA Methylation Status of the Estrogen Receptor $\alpha$ Gene in Canine Mammary Tumors. Vet. Pathol. 2018, 55, 510-516. [CrossRef]

18. Ren, X.; Li, H.; Song, X.; Wu, Y.; Liu, Y. 5-Azacytidine treatment induces demethylation of DAPK1 and MGMT genes and inhibits growth in canine mammary gland tumor cells. OncoTargets Ther. 2018, 11, 2805-2813. [CrossRef]

19. Qiu, H.; Lin, D. Roles of DNA mutation in the coding region and DNA methylation in the $5^{\prime}$ flanking region of BRCA1 in canine mammary tumors. J. Vet. Med. Sci. 2016, 78, 943-949. [CrossRef]

20. Feinberg, A.P.; Ohlsson, R.; Henikoff, S. The epigenetic progenitor origin of human cancer. Nat. Rev. Genet. 2006, 7, 21-33. [CrossRef]

21. Perakis, S.; Speicher, M.R. Emerging concepts in liquid biopsies. BMC Med. 2017, 15, 75. [CrossRef]

22. Board, R.E.; Knight, L.; Greystoke, A.; Blackhall, F.H.; Hughes, A.; Dive, C.; Ranson, M. DNA Methylation in Circulating Tumour DNA as a Biomarker for Cancer. Biomark. Insights 2007, 2, 307-319. [CrossRef]

23. Huang, J.; Wang, L. Cell-Free DNA Methylation Profiling Analysis-Technologies and Bioinformatics. Cancers 2019, 11, 1741. [CrossRef]

24. Constâncio, V.; Nunes, S.P.; Henrique, R.; Jerónimo, C. DNA Methylation-Based Testing in Liquid Biopsies as Detection and Prognostic Biomarkers for the Four Major Cancer Types. Cells 2020, 9, 624. [CrossRef]

25. Lee, K.-H.; Shin, T.-J.; Kim, W.-H.; Cho, J.-Y. Methylation of LINE-1 in cell-free DNA serves as a liquid biopsy biomarker for human breast cancers and dog mammary tumors. Sci. Rep. 2019, 9, 175. [CrossRef]

26. Nam, A.-R.; Lee, K.-H.; Hwang, H.-J.; Schabort, J.J.; An, J.-H.; Won, S.-H.; Cho, J.-Y. Alternative methylation of intron motifs is associated with cancer-related gene expression in both canine mammary tumor and human breast cancer. Clin. Epigenet. 2020, 12, 1-15. [CrossRef]

27. Herman, J.G.; Graff, J.R.; Myohanen, S.; Nelkin, B.D.; Baylin, S.B. Methylation-specific PCR: A novel PCR assay for methylation status of CpG islands. Proc. Natl. Acad. Sci. USA 1996, 93, 9821-9826. [CrossRef]

28. Akirav, E.M.; Lebastchi, J.; Galvan, E.M.; Henegariu, O.; Akirav, M.; Ablamunits, V.; Lizardi, P.M.; Herold, K.C. Detection of cell death in diabetes using differentially methylated circulating DNA. Proc. Natl. Acad. Sci. USA 2011, 108, 19018-19023. [CrossRef]

29. Eissa, M.A.L.; Lerner, L.; Abdelfatah, E.; Shankar, N.; Canner, J.K.; Hasan, N.M.; Yaghoobi, V.; Huang, B.; Kerner, Z.; Takaesu, F.; et al. Promoter methylation of ADAMTS1 and BNC1 as potential biomarkers for early detection of pancreatic cancer in blood. Clin. Epigenet. 2019, 11, 1-10. [CrossRef]

30. Giannopoulou, L.; Mastoraki, S.; Buderath, P.; Strati, A.; Pavlakis, K.; Kasimir-Bauer, S.; Lianidou, E.S. ESR1 methylation in primary tumors and paired circulating tumor DNA of patients with high-grade serous ovarian cancer. Gynecol. Oncol. 2018, 150, 355-360. [CrossRef]

31. Giannopoulou, L.; Chebouti, I.; Pavlakis, K.; Kasimir-Bauer, S.; Lianidou, E. RASSF1A promoter methylation in high-grade serous ovarian cancer: A direct comparison study in primary tumors, adjacent morphologically tumor cell-free tissues and paired circulating tumor DNA. Oncotarget 2017, 8, 21429-21443. [CrossRef]

32. Díez-Villanueva, A.; Mallona, I.; Peinado, M.A. Wanderer, an interactive viewer to explore DNA methylation and gene expression data in human cancer. Epigenet. Chromatin 2015, 8, 1-8. [CrossRef]

33. Schabort, J.J.; Nam, A.R.; Lee, K.H.; Kim, S.W.; Lee, J.E.; Cho, J.Y. ANK2 Hypermethylation in Canine Mammary Tumors and Human Breast Cancer. Unpublished work. 2020.

34. Lambert, S.; Bennett, V. Postmitotic expression of ankyrinR and beta R-spectrin in discrete neuronal populations of the rat brain. J. Neurosci. 1993, 13, 3725-3735. [CrossRef]

35. Bourguignon, L.Y.; Zhu, H.; Shao, L.; Zhu, D.; Chen, Y.W. Rho-kinase (ROK) promotes CD44v(3,8-10)-ankyrin interaction and tumor cell migration in metastatic breast cancer cells. Cell Motil. Cytoskelet. 1999, 43, $269-287$. [CrossRef]

36. Zhu, D.; Bourguignon, L.Y. Interaction between CD44 and the repeat domain of ankyrin promotes hyaluronic acid-mediated ovarian tumor cell migration. J. Cell. Physiol. 2000, 183, 182-195. [CrossRef]

37. Chen, Y.; Löhr, J.-M.; Jesnowski, R. Inhibition of Ankyrin-B Expression Reduces Growth and Invasion of Human Pancreatic Ductal Adenocarcinoma. Pancreatology 2010, 10, 586-596. [CrossRef]

38. Liao, C.; Huang, X.; Gong, Y.; Lin, Q. Discovery of core genes in colorectal cancer by weighted gene co-expression network analysis. Oncol. Lett. 2019, 18, 3137-3149. [CrossRef]

39. Stein, L.; Rothschild, J.; Luce, J.; Cowell, J.K.; Thomas, G.; Bogdanova, T.I.; Tronko, M.D.; Hawthorn, L. Copy Number and Gene Expression Alterations in Radiation-Induced Papillary Thyroid Carcinoma from Chernobyl Pediatric Patients. Thyroid 2010, 20, 475-487. [CrossRef] 
40. Wu, Z.-H.; Zhou, T.; Sun, H.-Y. DNA methylation-based diagnostic and prognostic biomarkers of nasopharyngeal carcinoma patients. Medicine 2020, 99, e20682. [CrossRef]

41. Heddleston, J.M.; Li, Z.; Lathia, J.D.; Bao, S.; Hjelmeland, A.B.; Rich, J.N. Hypoxia inducible factors in cancer stem cells. Br. J. Cancer 2010, 102, 789-795. [CrossRef]

42. Lau, K.W.; Tian, Y.-M.; Raval, R.R.; Ratcliffe, P.J.; Pugh, C.W. Target gene selectivity of hypoxia-inducible factor- $\alpha$ in renal cancer cells is conveyed by post-DNA-binding mechanisms. Br. J. Cancer 2007, 96, 1284-1292. [CrossRef]

43. Wallace, E.M.; Rizzi, J.P.; Han, G.; Wehn, P.M.; Cao, Z.; Du, X.; Cheng, T.; Czerwinski, R.M.; Dixon, D.D.; Goggin, B.S.; et al. A Small-Molecule Antagonist of HIF2 $\alpha$ Is Efficacious in Preclinical Models of Renal Cell Carcinoma. Cancer Res. 2016, 76, 5491-5500. [CrossRef]

44. Li, Z.; Bao, S.; Wu, Q.; Wang, H.; Eyler, C.; Sathornsumetee, S.; Shi, Q.; Cao, Y.; Lathia, J.; McLendon, R.E.; et al. Hypoxia-Inducible Factors Regulate Tumorigenic Capacity of Glioma Stem Cells. Cancer Cell 2009, 15, 501-513. [CrossRef]

45. Sun, H.-X.; Xu, Y.; Yang, X.-R.; Wang, W.-M.; Bai, H.; Shi, R.-Y.; Nayar, S.K.; Devbhandari, R.P.; He, Y.-Z.; Zhu, Q.; et al. Hypoxia inducible factor 2 alpha inhibits hepatocellular carcinoma growth through the transcription factor dimerization partner 3/ E2F transcription factor 1-dependent apoptotic pathway. Hepatology 2013, 57, 1088-1097. [CrossRef]

46. Imamura, T.; Kikuchi, H.; Herraiz, M.-T.; Park, D.-Y.; Mizukami, Y.; Mino-Kenduson, M.; Lynch, M.P.; Rueda, B.R.; Benita, Y.; Xavier, R.J.; et al. HIF- $1 \alpha$ and HIF- $2 \alpha$ have divergent roles in colon cancer. Int. J. Cancer 2009, 124, 763-771. [CrossRef]

47. Rawłuszko, A.A.; Horbacka, K.; Krokowicz, P.; Misztal, M.; Jagodziński, P.P. Prognostic Potential of DNA Methylation and Transcript Levels of HIF1A and EPAS1 in Colorectal Cancer. Mol. Cancer Res. 2014, 12, 1112-1127. [CrossRef]

48. Westerlund, I.; Shi, Y.; Toskas, K.; Fell, S.M.; Li, S.; Surova, O.; Södersten, E.; Kogner, P.; Nyman, U.; Schlisio, S.; et al. Combined epigenetic and differentiation-based treatment inhibits neuroblastoma tumor growth and links HIF2 $\alpha$ to tumor suppression. Proc. Natl. Acad. Sci. USA 2017, 114, E6137-E6146. [CrossRef]

49. Klahan, S.; Wong, H.S.-C.; Tu, S.-H.; Chou, W.-H.; Zhang, Y.; Ho, T.-F.; Liu, C.-Y.; Yih, S.-Y.; Lu, H.-F.; Chen, S.C.-C.; et al. Identification of genes and pathways related to lymphovascular invasion in breast cancer patients: A bioinformatics analysis of gene expression profiles. Tumor Biol. 2017, 39. [CrossRef]

50. Fuady, J.H.; Gutsche, K.; Santambrogio, S.; Varga, Z.; Hoogewijs, D.; Wenger, R.H. Estrogen-dependent downregulation of hypoxia-inducible factor (HIF)- $2 \alpha$ in invasive breast cancer cells. Oncotarget 2016, 7, 31153-31165. [CrossRef]

51. Jarman, E.J.; Ward, C.; Turnbull, A.K.; Martínez-Pérez, C.; Meehan, J.; Xintaropoulou, C.; Sims, A.H.; Langdon, S.P. HER2 regulates HIF-2 $\alpha$ and drives an increased hypoxic response in breast cancer. Breast Cancer Res. 2019, 21, 10. [CrossRef]

52. Xu, X.-H.; Bao, Y.; Wang, X.; Yan, F.; Guo, S.; Ma, Y.; Xu, D.; Jin, L.; Xu, J.; Wang, J. Hypoxic-stabilized EPAS1 proteins transactivate DNMT1 and cause promoter hypermethylation and transcription inhibition of EPAS1 in non-small cell lung cancer. FASEB J. 2018, 32, 6694-6705. [CrossRef]

53. Pan, R.; Zhou, C.; Dai, J.; Ying, X.; Yu, H.; Zhong, J.; Zhang, Y.; Wu, B.; Mao, Y.; Wu, D.; et al. Endothelial PAS domain protein 1 gene hypomethylation is associated with colorectal cancer in Han Chinese. Exp. Ther. Med. 2018, 16, 4983-4990. [CrossRef]

54. Kent, W.J.; Sugnet, C.W.; Furey, T.S.; Roskin, K.M.; Pringle, T.H.; Zahler, A.M.; Haussler, D. The human genome browser at UCSC. Genome Res. 2002, 12, 996-1006.

55. Győrffy, B.; Lanczky, A.; Eklund, A.C.; Denkert, C.; Budczies, J.; Li, Q.; Szallasi, Z. An online survival analysis tool to rapidly assess the effect of 22,277 genes on breast cancer prognosis using microarray data of 1809 patients. Breast Cancer Res. Treat. 2010, 123, 725-731. [CrossRef]

Publisher's Note: MDPI stays neutral with regard to jurisdictional claims in published maps and institutional affiliations. 